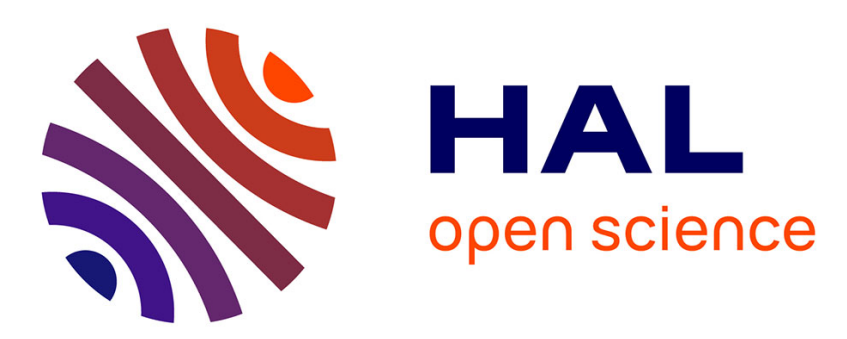

\title{
SOUND PROPAGATION IN RANDOM MEDIA. Backscattering correction to the multiple forward scattering theory
}

B. Li, R. Grosse, V. Mellert

\section{- To cite this version:}

B. Li, R. Grosse, V. Mellert. SOUND PROPAGATION IN RANDOM MEDIA. Backscattering correction to the multiple forward scattering theory. Journal de Physique IV Proceedings, 1992, 02 (C1), pp.C1-557-C1-560. 10.1051/jp4:19921120 . jpa-00251076

\section{HAL Id: jpa-00251076 https://hal.science/jpa-00251076}

Submitted on 1 Jan 1992

HAL is a multi-disciplinary open access archive for the deposit and dissemination of scientific research documents, whether they are published or not. The documents may come from teaching and research institutions in France or abroad, or from public or private research centers.
L'archive ouverte pluridisciplinaire HAL, est destinée au dépôt et à la diffusion de documents scientifiques de niveau recherche, publiés ou non, émanant des établissements d'enseignement et de recherche français ou étrangers, des laboratoires publics ou privés. 


\title{
SOUND PROPAGATION IN RANDOM MEDIA. Backscattering correction to the multiple forward scattering theory
}

\author{
B. LI, R. GROSSE and V. MELLERT \\ FB 8, Physik. Universität Oldenburg, Postfach 2503, D-2900 Oldenburg, Germany
}

\begin{abstract}
The Parabolic Equation Method (PEM) is the commonly used method to deal with wave propagation in random media. This method basically requires the small ratio of wave length to correlation length (small angle scattering). Recently, de Wolf/2/ and Rino/3/ extended the PEM by including the backscattered wave, retaining the small angle scattering. In his dissertation, Große/4/generalized the PEM to the case of forward wide angle scattering. In this paper, a further generalization leads to a solution for the first moment of the scalar Helmholtz equation without any restriction concerning the scattering angles.
\end{abstract}

\section{INTRODUCTION}

Sound propagating in the atmosphere often meets turbulent structures of the same size as the wave length. Therefore it is necessary to develope a theory without any restriction on the wave length. The commonly used Parabolic Equation Method /1/ basically requires the assumption of a small wave length to correlation length ratio. This means the restriction to small scattering angles. In recent papers, de Wolf/2/ and Rino/3/ extended the PEM by including the small angle backscattered wave. Große/4/ has generalized the Parabolic Equation Method to the case of forward wide angle scattering. In this paper both extensions of the PEM are combined to find a solution for the first statistical moment of the wave function without any restriction on the scattering angles. The result is found to be the same as obtained by the Diagramm Method $/ 5 /$.

\section{COUPLED MOMENT EQUATIONS}

Let us begin with the scalar Helmholtz equation:

$$
\left(\Delta+k^{2}(1+\mu(\vec{r}))\right) \psi(\vec{r})=0
$$

$\Delta$ is the Laplace operator, $\mathrm{k}$ the wave number and $\mu(\vec{r})$ the refractive index deviation from mean value. The random variable $\mu(\vec{r})$ has the following properties:

$$
<\mu(\vec{r})>=0, \quad \sqrt{<\mu^{2}>}=\epsilon \ll 1
$$

For the half-space problem one splits $\psi$ into two parts: the forward propagating wave $\psi^{+}$and the back propagating wave $\psi^{-}$. Equation (1) is equavilent to the following two integral equations:

$$
\begin{gathered}
\psi^{+}(\vec{\rho}, z)=\psi_{0}(\vec{\rho}, z)-k^{2} \int_{0}^{z} d z^{\prime} \int d^{2} \vec{\rho}^{\prime} G\left(\vec{r}, \vec{r}^{\prime}\right) \mu\left(\vec{r}^{\prime}\right) \psi\left(\vec{r}^{\prime}\right) \\
\psi^{-}(\vec{\rho}, z)=-k^{2} \int_{z}^{\infty} d z^{\prime} \int d^{2} \vec{\rho}^{\prime} G\left(\vec{r}, \vec{r}^{\prime}\right) \mu\left(\vec{r}^{\prime}\right) \psi\left(\vec{r}^{\prime}\right)
\end{gathered}
$$


$G\left(\vec{r}, \vec{r}^{\prime}\right)$ is the Greens function of the homogeneous Helmholtz equation and $z$ is the direction from the source to receiver.

To solve the coupled integral equations for the first statistical moment the Local Method of Small Perturbations (LMSP) which was developed by Beran/6,7/ is used. By this method the scattering volume is divided into slabs perpendicular to the $z$-direction. The thickness $\Delta z$ of each slab is chosen small enough to apply the Born approximation. In addition, we assume $\Delta z$ much bigger than the correlation length $l$ of the refractive index. Hence, subsequent slabs are statistical independent.

For the simplicity of mathematical representation, the following operators are introduced:

$$
\begin{gathered}
\mathcal{G}_{n}^{ \pm} \psi^{\mp}\left(\vec{r}_{n}\right)=\int d \vec{\rho}^{\prime} \int_{z_{n}}^{n \Delta z} d z^{\prime} G\left(\vec{r}_{n}, \vec{r}^{\prime}\right) \mu\left(\vec{r}^{\prime}\right) \psi^{\mp}\left(\vec{r}^{\prime}\right) d \vec{r}^{\prime} \\
\mathcal{G}_{n}^{ \pm} \psi^{ \pm}\left(\vec{r}_{n}\right)=\int d \vec{\rho} \int_{(n-1) \Delta z}^{z_{n}} d z^{\prime} G\left(\vec{r}_{n}, \vec{r}^{\prime}\right) \mu\left(\vec{r}^{\prime}\right) \psi^{ \pm}\left(\vec{r}^{\prime}\right) d \vec{r}^{\prime}
\end{gathered}
$$

Approximate to the second order in $\epsilon$, one obtains the following coupled iterative equations:

$$
\begin{gathered}
\psi_{n}^{+}=\left(1+\mathcal{G}_{n}^{+}+\mathcal{G}_{n}^{+} \mathcal{G}_{n}^{+}+\mathcal{G}_{n}^{-} \mathcal{G}_{n}^{-}\right) \psi_{n-1}^{+} \\
+\left(\mathcal{G}_{n}^{-}+\mathcal{G}_{n}^{+} \mathcal{G}_{n}^{-}+\mathcal{G}_{n}^{-} \mathcal{G}_{n}^{+}\right) \psi_{n}^{-} \\
\psi_{n-1}^{-}=\left(1+\mathcal{G}_{n}^{+}+\mathcal{G}_{n}^{+} \mathcal{G}_{n}^{+}+\mathcal{G}_{n}^{-} \mathcal{G}_{n}^{-}\right) \psi_{n}^{-} \\
+\left(\mathcal{G}_{n}^{-}+\mathcal{G}_{n}^{+} \mathcal{G}_{n}^{-}+\mathcal{G}_{n}^{-} \mathcal{G}_{n}^{+}\right) \psi_{n-1}^{+}
\end{gathered}
$$

Taking ensemble averages on eq.(7) and eq.(8) leads to moment equations which are not closed. Because the subsequent slabs are not correlated, these equations can be closed. This results in:

$$
\begin{aligned}
& \left\langle\psi_{n}^{+}\right\rangle=\left(1+\left\langle\mathcal{G}_{n}^{-} \mathcal{G}_{n+1}^{-}\right\rangle\right)\left\langle\psi_{n-1}^{+}\right\rangle+\left\langle\mathcal{G}_{n}^{+} \mathcal{G}_{n-1}^{-}\right\rangle\left\langle\psi_{n}^{-}\right\rangle \\
& +\left(\left\langle\mathcal{G}_{n}^{+} \mathcal{G}_{n-1}^{+}\right\rangle+\left\langle\mathcal{G}_{n}^{+} \mathcal{G}_{n}^{+}\right\rangle+\left\langle\mathcal{G}_{n}^{-} \mathcal{G}_{n}^{-}\right\rangle\right)\left\langle\psi_{n-2}^{+}\right\rangle \\
& +\left(\left\langle\mathcal{G}_{n}^{+} \mathcal{G}_{n}^{-}\right\rangle+\left\langle\mathcal{G}_{n}^{-} \mathcal{G}_{n}^{+}\right\rangle+\left\langle\mathcal{G}_{n}^{-} \mathcal{G}_{n+1}^{+}\right\rangle\right)\left\langle\psi_{n+1}^{-}\right\rangle \\
& \left\langle\psi_{n-1}^{-}\right\rangle=\left(1+\left\langle\mathcal{G}_{n}^{-} \mathcal{G}_{n-1}^{-}\right\rangle\right)\left\langle\psi_{n}^{-}\right\rangle+\left\langle\mathcal{G}_{n}^{+} \mathcal{G}_{n+1}^{-}\right\rangle\left\langle\psi_{n-1}^{+}\right\rangle \\
& +\left(\left\langle\mathcal{G}_{n}^{+} \mathcal{G}_{n+1}^{+}\right\rangle+\left\langle\mathcal{G}_{n}^{+} \mathcal{G}_{n}^{+}\right\rangle+\left\langle\mathcal{G}_{n}^{-} \mathcal{G}_{n}^{-}\right\rangle\right)\left\langle\psi_{n+1}^{-}\right\rangle \\
& +\left(\left\langle\mathcal{G}_{n}^{+} \mathcal{G}_{n}^{-}\right\rangle+\left\langle\mathcal{G}_{n}^{-} \mathcal{G}_{n}^{+}\right\rangle+\left\langle\mathcal{G}_{n}^{-} \mathcal{G}_{n-1}^{+}\right\rangle\right)\left\langle\psi_{n-2}^{+}\right\rangle
\end{aligned}
$$

\section{SOLUTION OF THE COUPLED EQUATIONS}

For the case of a statistical homogeneous medium, all terms in eq.(9) and eq.(10) become convolution products with respect to the variable $\vec{\rho}$. Hence, it is convenient to solve them in Fourier spectral domain. The 2-dimensional Fourier Transform is defined as:

$$
\mathcal{F}_{\rho}(f(\vec{\rho}, z))=\tilde{f}(\vec{\kappa}, z)=\frac{1}{(2 \pi)^{2}} \int d^{2} \vec{\rho} e^{i \vec{k} \cdot \vec{\rho}} f(\vec{\rho}, z)
$$

According to the Local Method of Small Perturbations, the mean values of the wave functions do not change significantly within one slab. Therefore, one can pass to an infinitesimal description by using the following limit/8/:

$$
\lim _{\Delta z \rightarrow 0, \frac{\Delta \pm}{l}, f i x e d} \frac{\left\langle\psi_{n}^{ \pm}(n \Delta z)>-<\psi_{n-1}^{ \pm}((n-1) \Delta z)\right.}{\Delta z} \approx \frac{\left.\partial<\psi^{ \pm}\right\rangle}{\partial z}
$$

After some mathematical calculations one can obtain:

$$
\frac{\partial<\tilde{\psi}^{+}>}{\partial z}=\left(i a-\sigma_{t}\right)<\tilde{\psi}^{+}>-\sigma_{t}<\tilde{\psi}^{-}>
$$




$$
-\frac{\partial<\tilde{\psi}^{-}>}{\partial z}=\left(i a-\sigma_{t}\right)<\tilde{\psi}^{-}>-\sigma_{t}<\tilde{\psi}^{+}>
$$

where: $\sigma_{t}=\sigma^{+}+\sigma^{-}$

$$
\sigma^{ \pm}=\frac{\pi k^{4}}{4} \int \frac{\bar{\phi}\left(\vec{\kappa}^{\prime}, a_{-} \mp a\right)}{a_{-} a} d^{2} \vec{\kappa}^{\prime}, \quad a=\sqrt{k^{2}-\vec{\kappa}^{2}}, \quad a_{-}=\sqrt{k^{2}-\left(\vec{\kappa}-\vec{\kappa}^{\prime}\right)^{2}}
$$

$\sigma_{t}, \sigma^{+}$and $\sigma^{-}$represent the total scattering, the forward scattering and the backscattering cross section per unit scattering volume, respectively. The $\tilde{\phi}\left(\vec{\kappa}^{\prime}, a\right)$ is the power spectrum of the refractive index field. Because of $a$ being a complex number, $\phi$ is defined as complex Laplace transform of the autocorrelation function ${ }^{1} B$.

For the special case that the random medium occupies the space with length of $L$ in $z$-direction, one can get a special solution of eq.(13) and eq.(14) by using the boundary conditons at $z=0$ and $z=L$ :

$$
\begin{aligned}
& <\tilde{\psi}^{+}(z)>=J\left(e^{i \lambda z}-\gamma^{2} e^{i \lambda(2 L-z)}\right)<\tilde{\psi}_{i n c}(0)> \\
& <\tilde{\psi}^{-}(z)>=J \gamma\left(e^{i \lambda z}-e^{i \lambda(2 L-z)}\right)<\tilde{\psi}_{i n c}(0)>
\end{aligned}
$$

where:

$$
\lambda=\sqrt{a^{2}+2 i a \sigma_{t}}, \quad J=\frac{1}{1-\gamma^{2} e^{2 i \lambda L}}, \quad \gamma=\frac{i a-\sigma_{t}-i \lambda}{\sigma_{t}}
$$

$<\tilde{\psi}_{\text {inc }}(0)>$ is the incident wave at boundary $z=0$.

\section{COMPARISON WITH OTHER METHODS}

\section{a. Comparison with Diagram Method}

The Diagram Method is the general theory for waves in weakly irregular random media. In order to compare our method with Diagram Method, the incident wave is assumed to be a plane wave and $L \rightarrow \infty$. Under this conditions, the results eq.(16) and eq.(17) can be transformed into: ${ }^{2}$

$$
\left\langle\psi^{+}(\vec{\rho}, z)\right\rangle=e^{i \lambda_{0} z}, \quad\left\langle\psi^{-}(\vec{\rho}, z)\right\rangle=\gamma_{0} e^{i \lambda_{0} z}
$$

where: $\quad \lambda_{0}=k \sqrt{1+\frac{2 i \sigma_{t_{2}}}{k}} \approx k+i \sigma_{t_{0}}, \quad\left|\frac{\sigma_{t_{0}}}{k}\right| \ll 1$.

The last approximation is valid for weakly irregular media and is consistent with the Local Method of Small Perturbations. If, in addition, the random medium is assumed to be isotropic and the correlation function is an exponential function, then:

$$
\sigma_{t_{0}}=-\frac{k^{3} l^{2}<\mu^{2} \geq}{2}\left(\frac{i-2 k l}{1+4(k l)^{2}}\right)
$$

This coefficient is exactly the one which is calculated by the Diagram Method/5/.

\section{b. Comparison with the Forward Scattering Theory(FST)}

In the Forward Scattering Theory/4/, the backscattering is neglected, i.e. $\left|\sigma_{b}\right| \ll\left|\sigma_{f}\right|$ thus $\sigma_{t} \approx \sigma_{f}$ and $\lambda$ becomes $\lambda_{0}=k+i \sigma_{f_{0}}$. This leads to the result obtained by FST/4/:

$$
\sigma_{t_{0}} \approx \sigma_{f_{0}}=\frac{k^{2} l<\mu^{2}>}{4}\left(\frac{k^{2} l^{2}-i k l}{1+k^{2} l^{2}}\right)
$$

\section{c. Comparison with Rino's result}

Rino's/3/ extension of PEM includes the backscattered wave. However, his results are restricted to small scattering angles. The results of eq.(16) and eq.(17) are valid for any scattering angle. Therefore, they

\footnotetext{
'The complex Laplace transform is defined as: $\dot{\phi}(\vec{\kappa}, a)=\frac{1}{\pi} \int_{0}^{\infty} \dot{B}(\vec{\kappa}, z) e^{i a x} d z$

${ }^{2}$ The subscripts of the parameters in following equations represent the corresponding parameters of the plane wave.
} 
can be reduced to Rino's results by evoking the approximation of a small wave length to correlation length ratio. Doing this, $a$ and $a_{-}$become approximately $k$. This substitution results in:

$$
\sigma_{t}=\frac{\pi k^{2}}{4} \int\left(\tilde{\phi}\left(\vec{\kappa}^{\prime}, 0\right)+\tilde{\phi}\left(\vec{\kappa}^{\prime}, 2 k\right)\right) d^{2} \vec{\kappa}^{\prime}
$$

which is the result obtained by Rino.

\section{CONCLUSIONS}

The Local Method of Small Perturbation has been used in this paper to deal with wave propagation in a random medium. By using this method, one can obtain the closed coupled equations (13) and (14), including both, forward- and backscattered waves. Solutions of these equations have no restrictions concerning the wave length to correlation length ratio.

In comparison with other commonly used methods, our generalized results show corrections to scattering cross section. Special results of Forward Scattering Theory, Rino's method and the Parabolic Equation Method can be deduced from our results by using corresponding approximations.

The method proposed in this paper is equivalent to the Diagram Method for the first statistical moment. Applying this method to the second statistical moment of the wave function perhaps give us a way of solving the Bethe-Salpether equation.

\section{ACKNOWLEDGEMENT}

The authors wish to thank Prof. Dr. K. Haubold, Dr. M. Schultz-von Glahn and A. Sill for the valuble discussions during the whole work. Baowen $\mathrm{Li}$ wishes to thank Prof. Z. W. Qian for the helpful suggestions and discussions through the private communications. Baowen $\mathrm{Li}$ is supported by the MaxPlanck-Gesellschaft.

\section{REFERENCES}

/1/. Rytov, S. M., Kartsov, Yu. A., Tatarskii, V. I. Principles of statistical Radiophysics: vol.4 Wave propagation through Random media. Springer-Verlag. Berlin-Heidelberg 1989.

12\% de Wolf, D. A. Backscatter Correction to the Parabolic Wave Equation. J. Opt. Soc. Am. 6(2) 1989. pp. 174-179.

13/. Rino, C. L. On propagation in continuous random media WAVES IN RANDOM MEDIA 2 (1991) pp. $59-72$

14/. Große, R., Ausbreitung von Wellen in Stochastischen Medien - Eine Verallgemeinerung der Parabolischen Näherung auf größere Wellenlängen. Dissertation, Carl-Von-Ossietzky Universität Oldenburg. Ocktober 1991.

15/. Bourret R. C. Stochastically Perturbed Fields, with Applications to Wave Propagation in Random Media Nuovo Cimento 26(1962) pp1-31

16/. Beran, M. J. Propagation of the mutual coherence Function through random media J. Opt. Soc. Am. 56(1966), pp. 1475-1480

/7/. ibid. 58(1968),pp.431-432

18/. Jr. Brown, W.P. Moment Equation for wave Propagated in Random Media J. Opt. Soc. Am. 62(1972), pp45-54. 